\title{
On the Energy and Centrality Dependence of Higher Order Moments of Net-Proton Distributions in Relativistic Heavy Ion Collisions
}

\author{
X. Wang and C. B. Yang \\ Institute of Particle Physics, Central China Normal University, Wuhan 430079, People's Republic of China and \\ Key Laboratory of Quark and Lepton Physics (CCNU), \\ Ministry of Education, People's Republic of China
}

(Dated: November 17, 2018)

\begin{abstract}
The higher order moments of the net-baryon distributions in relativistic heavy ion collisions are useful probes for the QCD critical point and fluctuations. Within a simple model we study the colliding energy and centrality dependence of the net-proton distributions in the central rapidity region. The model is based on considering the baryon stopping and pair production effects in the processes. Based on some physical reasoning, the dependence is parameterized. Predictions for the net-proton distributions for $\mathrm{Au}+\mathrm{Au}$ and $\mathrm{Pb}+\mathrm{Pb}$ collisions at different centralities at $\sqrt{s_{N N}}=39$ and $2760 \mathrm{GeV}$, respectively, are presented from the parameterizations for the model parameters. A possible test of our model is proposed from investigating the net-proton distributions in the non-central rapidity region for different colliding centralities and energies.
\end{abstract}

PACS numbers: 25.75.Gz, 21.65.Qr

\section{INTRODUCTION}

The investigation of QCD phase diagram is of crucial importance for our understanding of the properties of matter with strong interactions. Lattice QCD calculations have predicted, at vanishing baryon chemical potential, the occurrence of a cross-over from hadronic phase to the deconfined quark-gluon plasma phase above a critical temperature of about 170-190 MeV [1, 2]. A distinct singular feature of the phase diagram is the QCD critical point [3] which is located at the end of the transition boundary. A characteristic feature of the QCD critical point for systems in the thermodynamical limit is the divergence of the correlation length $\xi$ and extremely large critical fluctuations. In ultra-relativistic heavy ion collisions, however, because of finite size and rapid expansion of the produced system, those divergence may be washed out. As estimated in [3] , the critical correlation length in heavy ion collisions is not divergent but only about 2-3 fm. So the signals for the critical point of the system produced in heavy ion collisions cannot be observed as clearly as in the condensed matter physics. However, remnants of those critical large fluctuations may become accessible in heavy ion collisions through an event-by-event analysis of fluctuations in various channels of conservative hadron quantum numbers, for example, baryon number, electric charge, and strangeness [4]. Particularly there would be a non-monotonic behavior of non-Gaussian multiplicity fluctuations in an energy scan, which would be a clear signature for the existence of a critical point. In fact, at vanishing chemical potential it has been shown that moments of conservative charge distributions are sensitive indicators for the occurrence of a transition from hadronic to partonic matter [5].

Recently, great interest both experimentally [6] and theoretically [7-9] has been aroused on the higher order moments of net-baryon distributions in heavy ion collisions at the BNL Relativistic Heavy Ion Collider (RHIC) energies. The theoretical interest on these higher order moments comes from the discovery of the relation between the moments and the thermal fluctuations near the critical points for the produced quark matter. If some memory of the large correlation length in the quark matter sate persists in the thermal medium in hadronization process, this must be reflected in higher order moments of the distributions. Theoretical prediction [11] showed that the third moment, called skewness, is proportional to $\xi^{4.5}$ and that the fourth moment, or kurtosis, proportional to $\xi^{7}$ while the second moment proportional to $\xi^{2}$. More importantly, the moments are closely related to the susceptibilities of the thermal medium. Thus the higher order moments have stronger dependence on the correlation length $\xi$ and are therefore more sensitive to the critical fluctuations. Recently STAR Collaboration has published experimental data on the higher order moments [12] for different colliding systems at different colliding energies for different colliding centralities. It has been argued that the net-proton distribution can be a meaningful observable for the purpose of detecting the critical fluctuations of net baryons in heavy ion collisions [10]. This statement makes experimental investigation of netbaryon fluctuations much easier, because neutrons and strange baryons cannot been detected easily/effectively in experiments. Based on theoretical and experimental investigations, it has been argued that information of QCD phase diagram and the critical point can be obtained from the energy dependence of those moments [7].

The moments of net-proton distributions have been studied recently by quite a few groups with different event generators such as A Multi-Phase Transport (AMPT) and Ultrarelativistic Quantum Molecular Dynamics (UrQMD) 9], Heavy Ion Jet INteraction Generator (HIJING) 13], and hadron resonance gas model [14, 15] etc. Some other authors tried to search the statistical and dynamical components in the net-proton distributions in [16], where the statistical distributions for 
both proton and anti-proton are assumed Poissonian and the departure from Poisson distributions is regarded as the dynamical influence. One should pay attention that the use of independent Poisson distributions for proton and anti-proton implies that protons and anti-protons are produced completely uncorrelated. Therefore the baryon number conservation may be violated in any event. On the multiplicity distribution of hadrons, a canonical ensemble is employed in [17] to derive the number distribution for $\pi$ systems. This is reasonable because there are a lot of $\pi$ particles in the final state of heavy ion collisions. But a simple transportation of the method to the case for baryon production may be problematic, because the relevant baryon particle number may be not large enough for an equilibrium statistical description. In Ref. [18] the net-proton distributions in $\mathrm{Au}+\mathrm{Au}$ collisions at $\sqrt{s_{N N}}=200 \mathrm{GeV}$ are studied from very simple but well established physics considerations: baryon stopping and baryon pair production. For a given mean net-proton number from initial nuclear stopping, the initial proton number is assumed to satisfy a Poisson distribution. From the produced baryon pairs, the joint distribution for the newly produced proton and antiproton can be derived. Then one can obtain the netproton distribution in the final state of heavy ion collisions. Good agreement with experimental data has been obtained for $\mathrm{Au}+\mathrm{Au}$ collisions at three colliding centralities at $\sqrt{s_{N N}}=200 \mathrm{GeV}$.

This paper is an extension of the work in Ref. 18] by studying the moments of net-proton distributions in a given central rapidity window in $\mathrm{Au}+\mathrm{Au}$ collisions at lower RHIC energies at different centralities. This paper is organized as follows. In next section, we will address our model and the physics points for the centrality dependence of parameters. Using an analytical expressions for the net-proton distribution derived in Ref. [18] we will show that the moments of the distribution up to fourth order can all be well described by our model with suitably chosen parameters for four colliding energies in $\mathrm{Au}+\mathrm{Au}$ collisions at RHIC. In section III, our model results for the moments are compared with the experimental data from STAR Collaboration. The net-proton distributions for $\mathrm{Au}+\mathrm{Au}$ collisions at $\sqrt{s_{\mathrm{NN}}}=39 \mathrm{GeV}$ are presented for three centralities. Then in section IV, we discuss the energy dependence of the parameters and give parameterizations for such dependence. Then we extrapolate the dependence to the CERN Large Hadron Collider (LHC) energy and predict the net-proton distributions for $\mathrm{Pb}+\mathrm{Pb}$ collisions at different colliding centralities for $\sqrt{s_{\mathrm{NN}}}=2.76 \mathrm{TeV}$. The last section will be for a brief summary.

\section{MODEL CONSIDERATION FOR THE CENTRALITY DEPENDENCE}

Nuclear stopping plays an important role in heavy ion collisions and the study of such effect is a fundamental issue, since this effect is related to the amount of energy and baryon number that get transferred from the beam nucleons into the reaction zone. We denote $B$ the mean net-proton number in the final state distribution. As can be seen from our model consideration, $B$ comes only from the initially stopped protons. In nuclear-nuclear collisions the mean net-baryon number $B$ in central rapidity region would be zero if there were no nuclear stopping in the processes. Another physics point we consider in Ref. [18] and here is baryon pair production in the interactions. We denote $\mu$ the mean number of produced baryon pairs within a given kinematic region in the collisions at given colliding centrality. By assuming that baryon pairs are produced independently, the pair number distribution is of Poissonian. With isospin conservation, one can derive a simple analytical formula for the net-proton number $\Delta p$ distribution $P(\Delta p)$ as a function of $B$ and $\mu$ as [18]

$$
P(\Delta p)=\int_{0}^{\pi} \frac{d x}{\pi} e^{-(2 B+\mu) \sin ^{2} \frac{x}{2}} \cos (x \Delta p-B \sin x) .
$$

Now we study the centrality dependence of $B$ and $\mu$. Because the nuclear stopping effect results from the interactions between a passing nucleon with other nucleons on its way in a nuclear-nuclear collision, the baryon number stopped in a given kinematic region is closely related to the number of participant nucleons $N_{\mathrm{p}}$. In more central collisions the stopped net baryon number will be larger. In nuclear-nuclear collisions, if every nucleon from a nucleus suffers exactly the same interactions, the stopped proton number would be $B \propto N_{\mathrm{p}}$. Of course, the real case is not so simple. Because multiple scattering effect is more important for more central collisions, a little larger $B / N_{\mathrm{p}}$ can be expected for more central collisions. On the other hand, the stopped net-proton can be detected only in the final state, or in other words, after evolving with the system for some time. During the evolution of the system, the net-proton may diffuse into a kinematic region out of our interested window. For central collisions, the evolution time is longer and such diffusion effect is more obvious. This effect would reduce $B / N_{\mathrm{p}}$ for central collisions. The real proportional factor $B / N_{\mathrm{p}}$ is a result from the competition of the two effects: initial multiple scattering and later baryon number diffusing. Therefore, the factor $B / N_{\mathrm{p}}$ should have a weak centrality dependence. Thus one may parameterize the centrality dependence of $B$ as

$$
B=a_{1} N_{p}\left(1-a_{2} N_{p}\right),
$$

with $a_{1}$ and $a_{2}$ depending on the colliding energy of the system. While $a_{1}$ is always positive, the magnitude of $a_{2}$ should be small and $a_{2}$ can be negative or positive, depending on whether multiple scattering is more important than baryon number diffusion or the opposite. For the baryon pair production, similar physics considerations apply also. The probability for a baryon pair production near a point in the system is determined by the energy density at that point. If the energy density 


\begin{tabular}{||c|c|c|c|c||}
\hline \hline$\sqrt{s_{\mathrm{NN}}}(\mathrm{GeV})$ & $a_{1}$ & $10^{4} a_{2}$ & $b_{1}$ & $10^{4} b_{2}$ \\
\hline 19.6 & 0.022 & -0.126 & 0.0132 & 0.121 \\
\hline 39 & 0.0012 & -1.97 & 0.0300 & 4.90 \\
\hline 62.4 & 0.0096 & -0.159 & 0.0383 & 5.10 \\
\hline 200 & 0.0052 & 1.88 & 0.0585 & 5.00 \\
\hline \hline
\end{tabular}

TABLE I. Fitted parameters for four colliding energies.

of the hot strong interacting matter is uniform and the same for all colliding centralities, one may expect $\mu$ proportional to the volume of the system, thus $\mu \propto N_{\mathrm{p}}$. But the initial energy density is higher for more central collisions due to stronger nuclear stopping effect, a larger $\mu / N_{\mathrm{p}}$ is expected for more central collisions, if the nuclear stopping is the only physics in the process. On the other hand, as in the consideration for $B$, the energy diffusion from central to non-central rapidity region will reduce the value of $\mu / N_{\mathrm{p}}$. The competition of these two effect results in a behavior of $\mu$ as a function of $N_{\mathrm{p}}$ similar to that of $B$. Therefore we parameterize the colliding centrality dependence of $\mu$ as

$$
\mu=b_{1} N_{p}\left(1-b_{2} N_{p}\right)
$$

with $b_{1}$ and $b_{2}$ also depending on the colliding energy. As for $a_{2}$, the value of $b_{2}$ should be very small but can be positive or negative due to the competition of initial energy stopping and diffusion in the evolution of the system.

With the above four parameters, one can calculate the net-proton distribution and all the associate moments for any colliding centrality for given center of mass energy of the colliding system.

\section{COMPARISON WITH THE EXPERIMENTAL DATA}

For $\mathrm{Au}+\mathrm{Au}$ collisions at $\mathrm{RHIC}$ energies, we investigate the moments up to fourth order for the distribution of net-proton in the central rapidity window $|y|<0.5$ as functions of $N_{\mathrm{p}}$ by using our model described in the last section. The fitted results from our model for the moments are shown in Figs. 1-4 for the mean, variance, skewness and kurtosis for four colliding energies $\sqrt{s_{\mathrm{NN}}}=19.6,39,62.4$ and $200 \mathrm{GeV}$. The fitted parameters are tabulated in TABLE I. It can be seen that our simple model can describe quite well the centrality dependence of moments for the four energies with the parameters chosen. From the excellent agreement with the experimental data, one can conclude that our model contains the necessary physics for the net-proton distributions.

With those parameters in TABLE I one can calculate the net-proton distributions quite easily for different centralities for the four energies as in TABLE I Our new parametrization for $B$ and $\mu$ can give distributions for $\mathrm{Au}+\mathrm{Au}$ collisions at $\sqrt{s_{\mathrm{NN}}}=200 \mathrm{GeV}$. The newly obtained distributions have no visible difference from those

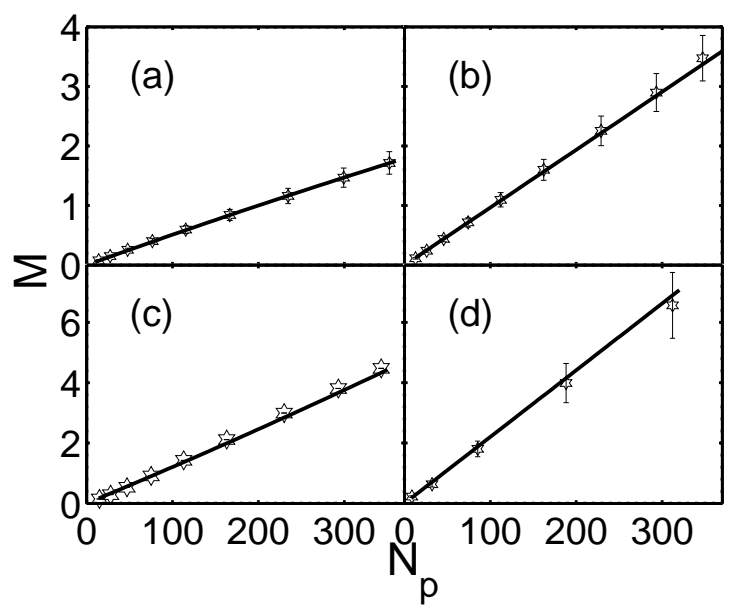

FIG. 1. Mean value of the net-proton distributions as a function of $N_{\mathrm{p}}$ for $\mathrm{Au}+\mathrm{Au}$ collisions at four $\sqrt{s_{\mathrm{NN}}}$, (a) 200; (b) 62.4 ; (c) 39 and (d) $19.6 \mathrm{GeV}$. The points are from RHIC/STAR data [12].

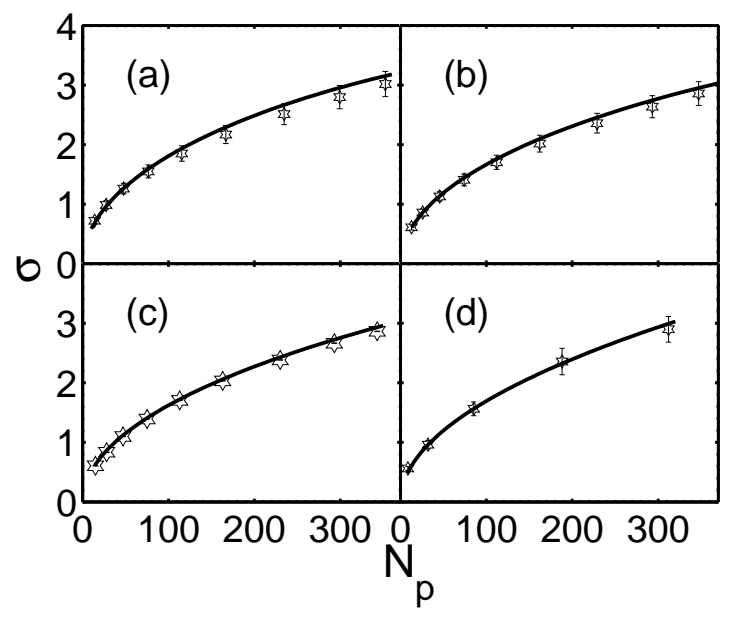

FIG. 2. variance of the net-proton distributions as a function of $N_{\mathrm{p}}$ for $\mathrm{Au}+\mathrm{Au}$ collisions at four $\sqrt{s_{\mathrm{NN}}}$, (a) 200; (b) 62.4; (c) 39 and (d) $19.6 \mathrm{GeV}$. The points are from RHIC/STAR data [12].

in Ref. [18], in good agreement with the STAR data, so will not be presented here. As an example to show the distributions, we present here only the distributions at $\sqrt{s_{\mathrm{NN}}}=39 \mathrm{GeV}$ for $\mathrm{Au}+\mathrm{Au}$ collisions at different colliding centralities, for later comparison with experimental results.

\section{COLLIDING ENERGY DEPENDENCE OF NET-PROTON DISTRIBUTION}

After discussing the net-proton distributions for $\mathrm{Au}+\mathrm{Au}$ collisions at four different colliding energies, one can discuss the colliding energy dependence of parameters in our model. As we discussed in Sec. II, the values and their dependence on the colliding energy can tell us 


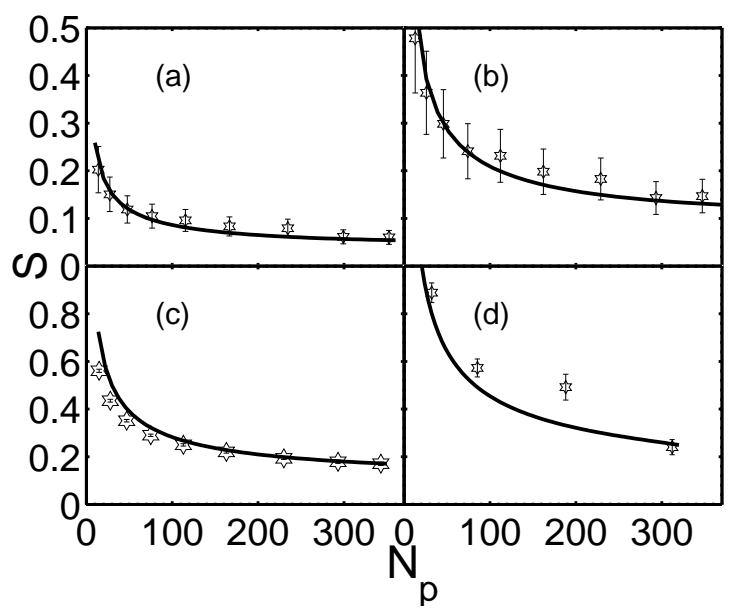

FIG. 3. Skewness of the net-proton distributions as a function of $N_{\mathrm{p}}$ for $\mathrm{Au}+\mathrm{Au}$ collisions at four $\sqrt{s_{\mathrm{NN}}}$, (a) 200; (b) 62.4 ; (c) 39 and (d) $19.6 \mathrm{GeV}$. The points are from RHIC/STAR data [12].

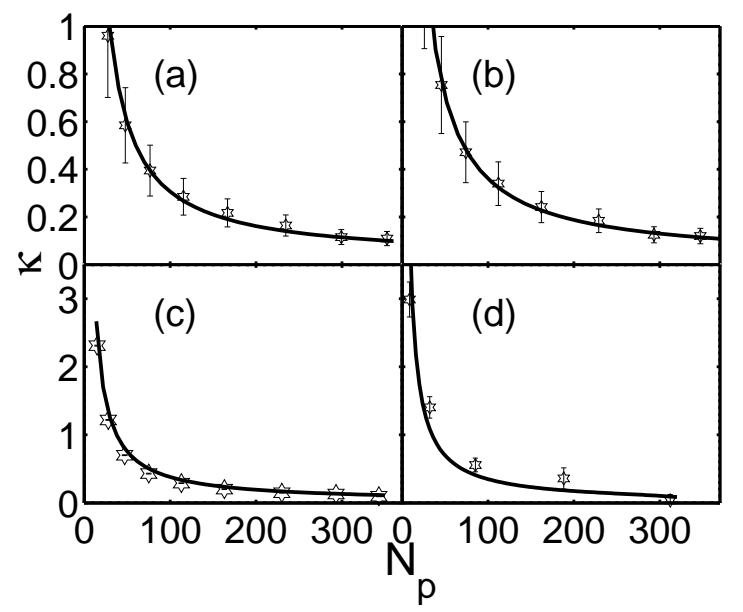

FIG. 4. Kurtosis of the net-proton distributions as a function of $N_{\mathrm{p}}$ for $\mathrm{Au}+\mathrm{Au}$ collisions at four $\sqrt{s_{\mathrm{NN}}}$, (a) 200; (b) 62.4; (c) 39 and (d) $19.6 \mathrm{GeV}$. The points are from RHIC/STAR data [12].

some physics in the colliding process, especially the competition of effects from the initial multiple scattering and later baryon number transportation. With the increase of colliding energy, the stopped baryon number in the central rapidity region will decrease. Thus the value of $a_{1}$ will decrease with $\sqrt{s_{\mathrm{NN}}}$. Though the energy fraction stopped in the central rapidity region becomes smaller at higher colliding energy, the energy density in that region still increases with $\sqrt{s_{\mathrm{NN}}}$. Then $b_{1}$ will increase with $\sqrt{s_{\mathrm{NN}}}$. For $\sqrt{s_{\mathrm{NN}}}$ high enough, one may expect $a_{1}$ and $b_{1}$ saturates at some limiting values. The behaviors of $a_{2}$ and $b_{2}$ can be quite different from those of $a_{1}$ and $b_{1}$. Since $a_{2}$ and $b_{2}$ depend on the competition of effects from initial multiple nucleon-nucleon scattering and later baryon/energy diffusion, the behaviors of $a_{2}$ and $b_{2}$ with

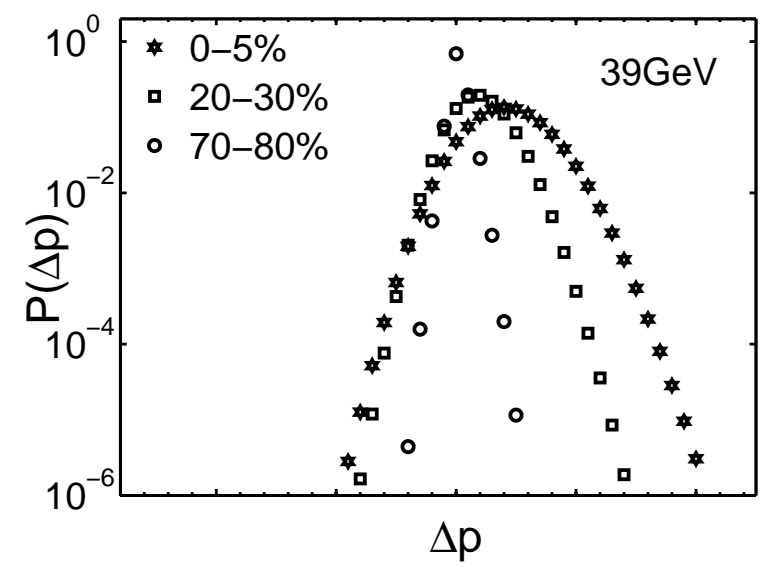

FIG. 5. Net-proton distributions for $\mathrm{Au}+\mathrm{Au}$ collisions at $\sqrt{s_{\mathrm{NN}}}=39 \mathrm{GeV}$ at different colliding centralities.

the increase of $\sqrt{s_{\mathrm{NN}}}$ can be complicated. Both the effects from the initial multiple nucleon-nucleon scattering and later baryon/energy diffusion become stronger with the increase of $\sqrt{s_{\mathrm{NN}}}$, but their rates of increase may be different. For a given increase of $\sqrt{s_{\mathrm{NN}}}$, if the initial multiple scattering becomes more important, $\left|a_{2}\right|\left(\left|b_{2}\right|\right)$ will increase with $\sqrt{s_{\mathrm{NN}}}$ in the region with negative $a_{2}\left(b_{2}\right)$. Otherwise, $\left|a_{2}\right|\left(\left|b_{2}\right|\right)$ will decrease in the same region. For $\sqrt{s_{\mathrm{NN}}} \rightarrow \infty$, the interaction duration in the produced matter will very long and the diffusion effect will be much stronger than that from initial multiple scattering. Then one can expect $a_{2}$ and $b_{2}$ approaching some positive saturating values, implying that $B / N_{\mathrm{p}}$ and $\mu / N_{\mathrm{p}}$ is smaller for central collisions when $\sqrt{s_{\mathrm{NN}}}$ is high enough.

To see the colliding energy dependence of the four parameters, we plot the fitted parameters shown in TABLE $\mathrm{I}$ as functions of $\sqrt{s_{\mathrm{NN}}}$ in $\mathrm{GeV}$. The plots are shown in Figs. 6] and 7. The shown dependence of the parameters on the colliding energy can be described by the following expressions

$$
\begin{aligned}
a_{1} & =14.55\left(1+9.23 \times 10^{-3} \sqrt{s_{\mathrm{NN}}}\right) /\left(1+40.2 \sqrt{s_{\mathrm{NN}}}\right), \\
10^{4} a_{2} & =1.87-1.21 \times 10^{-4}\left(\sqrt{s_{\mathrm{NN}}}\right)^{4} \exp \left(-0.107 \sqrt{s_{\mathrm{NN}}}\right), \\
b_{1} & =-0.02\left(1-0.105 \sqrt{s_{\mathrm{NN}}}\right) /\left(1+0.0295 \sqrt{s_{\mathrm{NN}}}\right), \\
10^{4} b_{2} & =5.0-0.626\left(\sqrt{s_{\mathrm{NN}}}\right)^{7.14} \exp \left(-0.979 \sqrt{s_{\mathrm{NN}}}\right) .
\end{aligned}
$$

The functional form for $a_{i}$ and $b_{i}(i=1,2)$ are chosen to satisfy the demands from the physics considerations in the last paragraph. With the above expressions for the energy dependence of the parameters, it is straightforward to calculate the values of those parameter at the LHC energy $\sqrt{s_{\mathrm{NN}}}=2760 \mathrm{GeV}$. By assuming that our model can be applied to that energy, one can calculate the net-proton distributions at that energy for different colliding centralities. The obtained distributions are shown in Fig. 8, The values of $N_{\mathrm{p}}$ used in the calculation are from [19].

In all considerations up to now, we only discussed the 


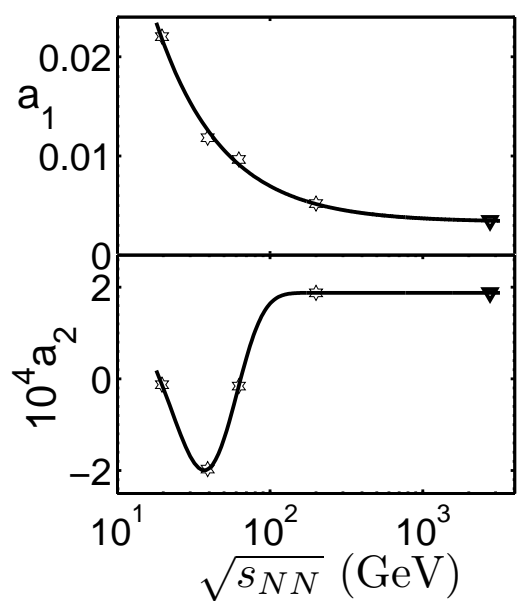

FIG. 6. Colliding energy dependence of parameters $a_{1}$ and $a_{2}$ for the initially stopped proton number in the given central rapidity window. Points marked by star are from our model fitting, and the points marked by triangle are calculated from Eq. (4) at $\sqrt{s_{\mathrm{NN}}}=2760 \mathrm{GeV}$.

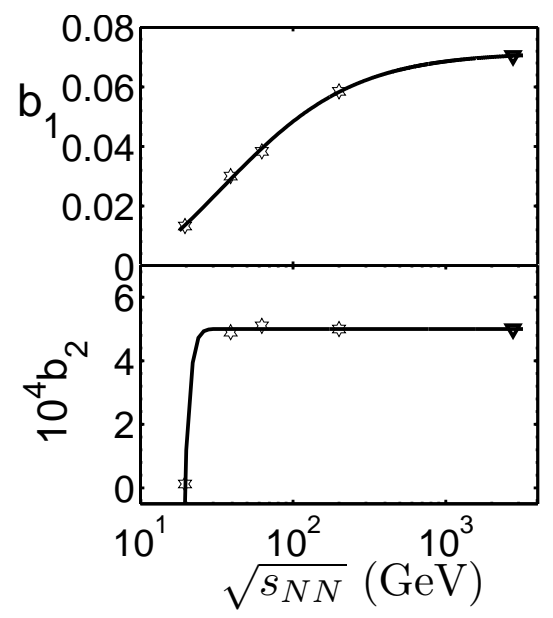

FIG. 7. Colliding energy dependence of parameters $b_{1}$ and $b_{2}$ for the mean number of produced baryon pairs in the given central rapidity window. Points marked by star are from our model fitting, and the points marked by triangle are calculated from Eq. (4) at $\sqrt{s_{\mathrm{NN}}}=2760 \mathrm{GeV}$.

net-proton distributions in the central rapidity region $|y|<0.5$. Because of the diffusion of baryon number and energy from central to non-central rapidity region, the parameters $a_{2}$ and $b_{2}$ show complicated behaviors as functions of colliding energy. If we consider the netproton distributions in the non-central region, $|y|>0.5$ for say, the same physics arguments apply and one can expect that the distributions can also be described by Eq. (1) with centrality dependence of parameters $B$ and $\mu$ being given by Eqs. (2) and (3). One can also expect that the colliding energy dependence of $a_{1}$ and $b_{1}$ is similar to that for the case in central rapidity region. For $a_{2}$ and $b_{2}$, the baryon number and energy diffusion from central to non-central region will not compete to but co-

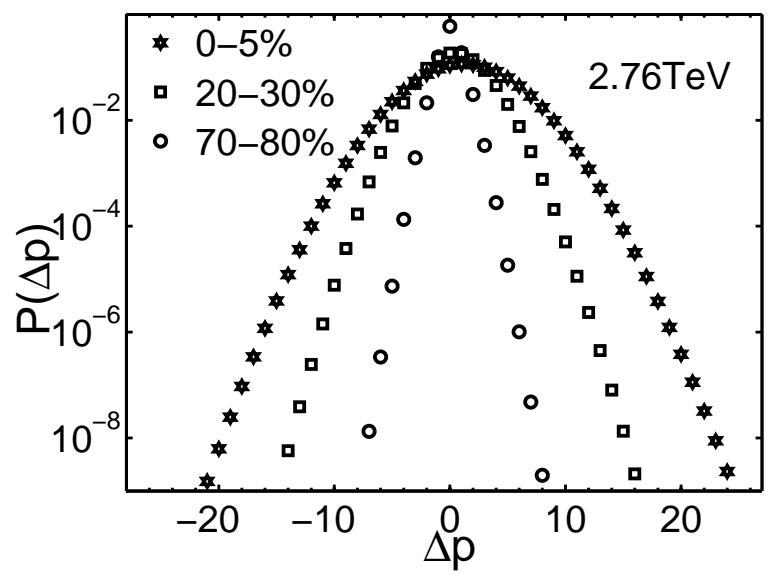

FIG. 8. Net-proton distributions for $\mathrm{Pb}+\mathrm{Pb}$ collisions at $\sqrt{s_{\mathrm{NN}}}=2760 \mathrm{GeV}$ at different colliding centralities.

operate with the multiple scattering effect. Then $a_{2}$ and $b_{2}$ will be negative for all colliding energies and all $\mathrm{A}+\mathrm{A}$ collisions. This statement can be tested experimentally.

\section{CONCLUSION}

We studied the net-proton distributions for $\mathrm{Au}+\mathrm{Au}$ collisions at four colliding energies for $\sqrt{s_{\mathrm{NN}}}$ from 19.6 to $200 \mathrm{GeV}$ at different centralities. Based on some physical arguments, the parameters in our model are parameterized as functions of centrality and energy. The higher order moments for the distributions are in good agreement with the experimental data. Prediction for the netproton distributions at LHC energy $\sqrt{s_{\mathrm{NN}}}=2.76 \mathrm{TeV}$ is presented for different centralities. The net-proton distribution in a non-central rapidity region and its dependence on centrality are discussed.

It should be mentioned that nothing else is assumed in this model except an initial stopped net-proton and a finite probability for producing baryon pairs from the produced matter. Therefore, our model has nothing to do with thermal equilibrium and/or critical fluctuations. Because our model consideration is based on normal physics effects, our results can be used as a baseline for detecting novel physics in the processes.

\section{ACKNOWLEDGMENTS}

This work was supported in part by the National Natural Science Foundation of China under Grant No. 11075061 and by the Programme of Introducing Talents of Discipline to Universities under No. B08033. The authors thank Dr. X.F. Luo for sending us the experimental data. We are grateful to N. Xu and X.F. Luo for valuable discussions. 
[1] Y. Aoki et al., Nature 443, 675 (2006); M. Cheng et al., Phys. Rev. D 74, 054507 (2006).

[2] J. Berges, K. Rajagopal, Nucl. Phys. B 538, 215 (1999).

[3] M. Stephanov, K. Rajagopal and E. Shuryak, Phys. Rev. D 60, 114028 (1999).

[4] M.A. Stephanov,K. Rajagopal and E.V. Shuryak, Phys. Rev. Lett. 81, 4816 (1998); S. Jeon, V. Koch, Phys. Rev. Lett. 85, 2076 (2000); M. Asakawa, U.W. Heinz and B. Müller, Nucl. Phys. A 698, 519 (2002); V. Koch, J. Phys. G 35, 104030 (2008).

[5] S. Ejiri, F. Karsch and K. Redlich, Phys. Lett. B 633, 275 (2006).

[6] M.M. Aggarwal et al., (STAR Collaboration), Phys. Rev. Lett. 105, 022302 (2010).

[7] S. Gupta et al., Science 332, 1525 (2011); X.F. Luo, B. Mohanty, H.G. Ritter and N. Xu, arXiv:1105.5049.

[8] F. Karsh and K. Redlich, Phys. Lett. B 695, 136 (2011); M. A. Stephanov, Phys. Rev. Lett. 107, 052301 (2011); B. Friman et al., Eur. Phys. J. C 71, 1694 (2011).

[9] Y. Zhou et al., Phys. Rev. C 82, 014905 (2010); K. Xiao et al., Chin. Phys. C 35, 467 (2011).

[10] Y. Hatta and M.A. Stephanov, Phys. Rev. Lett. 91, 102003 (2003).

[11] M.A. Stephanov, Phys. Rev. Lett. 102, 032301 (2009).

[12] X.F. Luo (for the STAR Collaboration), J. Phys: Conf. Ser. 316, 012003 (2011).

[13] X.F. Luo et al., J. Phys.G 37, 094061 (2010).

[14] P. Braun-Munzinger, K. Redlich and J. Stachel, in Quark Gluon Plasma 3, edited by R. C. Hwa and X.-N. Wang (World Scientific, Singapore, 2004); A. Andronic, P. Braun-Munzinger and J. Stachel, Nucl. Phys. A 772, 167 (2006).

[15] F. Karch and K. Redlich, Phys. Lett. B 695, 136 (2011).

[16] L.Z. Chen et al., J. Phys. G 38, 115005 (2011).

[17] V.V. Begun et al., Phys. Rev. C 70, 034901 (2004).

[18] C.B. Yang and X. Wang, Phys. Rev. C 84, 064908 (2011).

[19] K. Aamodt et al. (ALICE Collaboration), Phys. Rev. Lett. 106, 032301 (2011). 